\title{
A RAT SPINAL CORD INJURY EXPERIMENTAL MODEL
}

\author{
Šulla, I. ${ }^{1}$, Balik, V. ${ }^{2}$, Petrovičová, $\mathrm{J}^{3}$ \\ Almášiová, V. ${ }^{1}$, Holovská, K. ${ }^{1}$, Oroszová, Z. ${ }^{4}$ \\ ${ }^{1}$ Department of Anatomy, Histology and Physiology \\ University of Veterinary Medicine and Pharmacy, Komenského 73, 04181 Košice \\ The Slovak Republic \\ ${ }^{2}$ Department of Neurosugery, University Hospital, I. P. Pavlova 6, 77220 Olomouc \\ The Czech Republic \\ ${ }^{3}$ Department of Medical Informatics, Faculty of Medicine, P. J. Šafárik University \\ SNP 1, 04011 Košice, \\ ${ }^{4}$ Institute of Neurobiology, Slovak Academy of Sciences Šoltésovej 4, 04001 Košice \\ The Slovak Republic
}

igor.sulla@uvlf.sk

\section{ABSTRACT}

Spinal cord injuries (SCI) with their tragic consequences belong to the most serious pathological conditions. That is why they have stimulated basic research workers, as well as health care practitioners, to search for an effective treatment for decades. Animal experimental models have been essential in these efforts. We have jointly decided to test and standardize one of the spinal cord injury compression models in rats. Twentythree adult female Wistar rats weighing 250-320 g were utilized. Employing general anaesthesia along with a mixture of sevoflurane with $\mathrm{O}_{2}, 2$ rats (sham controls) had their vertebral arch of either Th8 or Th9 vertebra removed (laminectomy). The other 21 experimental rats with similar laminectomies were divided into 3 subgroups $(\mathbf{n}=7)$ which received compression impact forces of 30,40 or $50 \mathrm{~g}$ (subgroups-1, -2 , and -3 , respectively) applied on their exposed spinal medulla for 15 minutes. All rats were observed for 28 days after the experimental procedure and their motor functions were assessed by the Basso, Beattie, Bresnahan (BBB) test 6 hours, 7, 21 and 28 days after the simulated SCI. All 23 rats survived the surgical procedures. The control rats were without any neurological deficits. There were, in every experimental subgroup, 1 or 2 rats with extreme BBB scores. So the rats with the maximum and minimum $\mathrm{BBB}$ values were excluded. Then, the results acquired in the residual 5 rats in each group were averaged and statistically analysed by the Tukey multiple comparisons test. Statistically significant intersubgroup differences were found at all survival times equal to or longer than 7 post SCI days. The goal of the SCI experiment was to generate a reproducible and reliable, submaximal spinal cord trauma model. The statistical analyses demonstrated that this objective was best achieved in the subgroup- 2 with the $40 \mathrm{~g}$ compression.

Key words: compression model; rat; spinal cord trauma 


\section{INTRODUCTION}

A spinal cord injury (SCI), often produces extensive and persistent motor, and sensory deficits and vegetative states which causes a lifelong disability with major negative impacts on the patients, their families, as well as the society in general [3]. The current global estimates indicate that the incidence of SCI ranges from; 15 per million in Australia to 40 per million in the North America; and 14.7-40 per million in Europe (20 per million in Slovakia), with a mortality rate of 4.4-6.7\%, depending on the extent and location of the medullary lesions. The average age at injury is 31.7 years old with the greatest frequency between 15 and 25 years old and with the male to female ratio of $3: 1[4,7$, 18]. The surviving individuals from these SCI experiences, often are confined to a wheelchair and suffer considerable morbidity impacting on their quality of life $[2,4,18]$. The health care costs are significant with a single patient in the USA requiring between 1 and 25 million dollars over a lifetime depending on the severity of his/her injury [5]. So far, despite improvements in the medical and surgical management of SCI, there are no effective therapeutic measures able to improve neurological outcomes following spinal cord trauma. Only progress in neuroscience research, based on animal studies supported with gradable and qualifiable outcomes can hope to change this situation. We collectively decided to test and eventually modify the rat SCI compression model with the intention to standardise this model for further utilisation in planned SCI experiments.

\section{MATERIALS AND METHODS}

The experimental protocols were elaborated in compliance with the Animal Protection Act of the Slovak Republic No. 15/1995 [1] and approved by the Ethical Commission of the Institute of Neurobiology, Slovak Academy of Sciences in Košice, as well as by the State Veterinary Administration in Bratislava (decision No. 2812/13/221).

The basic experimental plan involved 23 adult female Wistar rats weighing 250-320 g. Two of the rats were kept as sham controls. Twenty-one rats were randomly divided into three subgroups of 7 animals each. A 15 minute compressing force of 30, 40 and $50 \mathrm{~g}$ (subgroups-1, -2, and -3, respectively) was applied directly on the intact dural sac containing the spinal medulla which had previously been exposed by the laminectomy of the 8th or 9th thoracic vertebra. More specific experimental details follow.

After the induction of general anaesthesia by vapours of isoflurane („Forane“, i.e. 1-chloro-2,2,2-trifluoroethyldifluoro-methylether, Abbott Laboratories Ltd., Queensborough, Great Britain) with $\mathrm{O} 2$ in a plexiglass box, all 23 rats were transferred to a heated platform, in order to maintain their rectal temperature between 36.6 and $37.2{ }^{\circ} \mathrm{C}$. The temperature was monitored directly by an inserted rectal thermometer probe. The inhalation anaesthesia (by a mixture of $1.5 \%$ isoflurane with medical oxygen) was delivered by a special mask designated for rodents.

The skin of the dorsal part of the rat's thoracic region was shaved and disinfected by iodine solution („Betadine“, i.e. povidonum iodatum, Egis Pharmaceuticals, Budapest, Hungary), and then the skin, subcutaneous fat and dorsal fascia were incised and an operating microscope Leica M320-F12 (Leica Microsystems CMS, GMbH, Herbrugg, Switzerland) was introduced. With the assistance of an improved illumination and magnification of tiny structures in the operational field provided by the microscope, the paravertebral muscles were separated from the spinous processes as well as the arches of Th8-Th10 vertebrae using microinstruments.

The spinal dural sac containing the spinal medulla was uncovered by laminectomy of either the 8th or 9th thoracic vertebra. In order to enhance the orientation of the operational field, a line connecting both caudal angles of the scapulae, which crossed the spinous process of the Th7 vertebra in a rat was used [10]. With an aim to prevent the constant movements of the spine and spinal cord caused by breathing of the rat, its vertebral column was suspended and fixed to a stereotaxic frame by Kocher clamps attached to the spinous processes of the 7 th and 10 th vertebrae, or by sutures pulled through the interspinous ligaments.

After a 15 minute exposure of the spinal dural sac to the surrounding environment, the procedure in the control rats were finished by sutures of the surgical wounds. The spinal cord trauma (SCI) to the 21 experimental rats was performed by a steel rod (freely moving up and down in a tube with minimal friction). At the distal end of the rod was a circular impactor with a diameter of $2 \mathrm{~mm}$ made of inert material which was placed directly on the exposed spinal dural sac. The metallic rod together with the impactor weighed $10 \mathrm{~g}$. On the top of the rod were placed various weights so that the impactor acted on the spinal cord by an exact compression force of 30, 40 or $50 \mathrm{~g}$ for 15 minutes (Fig. 1). 
After the spinal cord compression, the metallic rod with the impactor was removed and the operational opening was sutured in anatomical layers. Following laminectomy (in 2 sham controls), and the execution of spinal cord compression trauma (in 21 rats), all rats were administered antibiotics ("Amoksiklav", i. e. amoxicillinum natricum + kalii clavulans, Sandoz Pharmaceuticals d.d., Ljubljana, Slovenia) intramuscularly in a total dose of $30 \mathrm{mg}$. Then, the rats were transferred to disinfected cages, where they were provided with drinking water ad libitum and food granules. The 21 rats belonging to the experimental subgroups were prepared for studies of different aspects of the complex problem of SCI or for testing various therapeutic measures aimed to reduce the extent of the spinal cord damage and neurological deficits.

The rats were observed for 28 days. During this period their general condition, activity, neurological state, healing of surgical wounds and their motor functions by an open field Basso, Beattie, Bresnahan (BBB) test [2] were evaluated by two independent observers 6 hours following the SCI, when they completely recovered from anaesthesia, and then again at the 7 th, 14th, 21st and 28th post-SCI day. The maximal and minimal values of the BBB scores obtained from the experimental rats in each subgroup were considered coincidental, so they were left out; then, behavioural data from the five remaining rats belonging to each subgroup were averaged and the intergroup differences analysed statistically by the Tukey multiple comparisons test.

\section{RESULTS}

\section{Recovery of rats}

The two control rats survived their laminectomies and the 21 experimental rats survived their laminectomies and the compression of the spinal cord trauma. Both the controls and the experimental groups survived the 28-days observation period. Urinary retention developed in only 2 rats from subgroup-2 with medullary lesions caused by the $40 \mathrm{~g}$ compression force but in 6 from the subgroup- 3 (50 g compression). The retention lasted for 2-5 days, when their urinary bladder had to be emptied by Credés manoeuvre (manual bladder expression) every 12 hours. Later on, the ability to void spontaneously returned, even in the severely paraparetic or paraplegic rats. The surgical wounds were completely healed in all 23 rats on the 7th day following the surgery.

\section{Behavioural outcomes}

In both of the rats from the control group, their BBB score was 21 points (i.e. they did not exhibit any neurological deficits) 6 hours after the surgery.

The SCI rats from subgroup-1 (30 g compression) were paraplegic or severely paraparetic (BBB scores ranged from 0 to 2 points with an average value of 1.6 points) after the surgery. All of the rats from subgroup-2 ( $40 \mathrm{~g}$ compression) were paraplegic (BBB score 0$)$ after the surgery. Five rats from subgroup-3 (50 g compression) were paraplegic (BBB

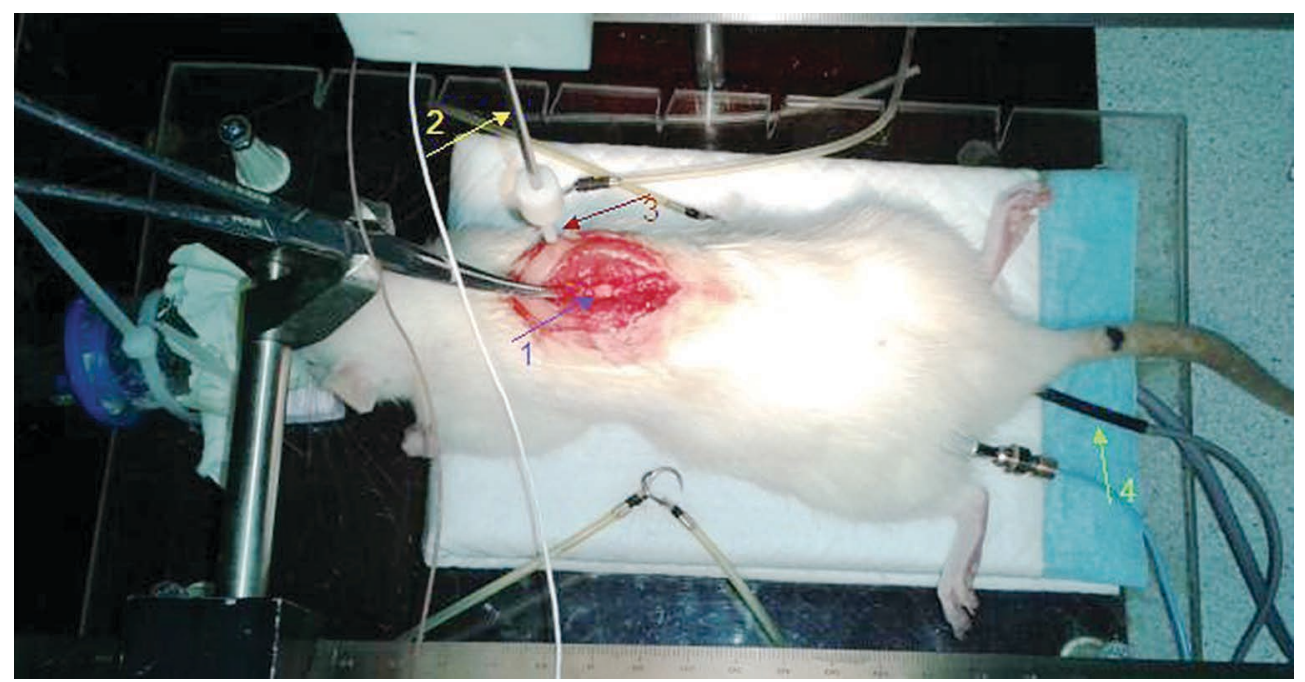

Fig. 1. Situation following laminectomy of Th9 vertebra in one of the experimental rats. The spinal dural sac containing the medulla spinalis is marked by the arrow No 1 . The spinous process of the Th7 is fixed by a Kocher clamp. The metallic rod (arrow No. 2) with an impactor (arrow No. 3 ) is visible cranially from the laminectomy to enable fixation of the spinous process of the Th10 by another Kocher clamp before the execution of spinal cord compression trauma. A thermometer probe (arrow No. 4) is inserted into the rectum of the rat 
score 0 ) after the surgery. The statistical analyses (by the Tukey multiple comparisons test) revealed no significant differences in the BBB scores among rats from all of the 3 experimental subgroups.

Seven days after the SCI, the BBB score in 5 rats from subgroup-1 was $6-7$ points (an average value of 6.6 points), in subgroup-2, it was $1-5$ points (average of 3.4 points) and in subgroup-3 it was $1-3$ points (average of 2.2 points). The statistical analyses showed a highly significant difference in the improvement of motor functions (expressed by $\mathrm{BBB}$ score) in rats belonging to the subgroup-1 versus rats belonging to the subgroup-3 $(\mathrm{P}=0.0001)$ and a significant difference between rats belonging to the subgroup-1 and rats belonging to the subgroup- $2(\mathrm{P}=0.002)$. There was no significant difference between rats belonging to the subgroup-2 and rats from the subgroup-3 $(\mathrm{P}=0.2502)$.

Fourteen days after the SCI, the BBB score in 5 rats from subgroup-1 was $7-8$ points (an average value of 7.6 points), in subgroup-2, it was $2-7$ points (an average value of 5.2 points) and in subgroup-3, it was $2-3$ points (average of 2.6 points). The statistical analyses of the results showed a highly significant difference in the improvement of motor functions (BBB score) between rats belonging to the subgroup- 1 versus rats belonging to the subgroup- 3 $(\mathrm{P}=0.0001)$, a significant difference between rats belonging to the subgroup-2, versus rats belonging to the subgroup-3 $(\mathrm{P}=0.017)$, and in rats belonging to the subgroup 1 , versus rats belonging to the subgroup-2 $(\mathrm{P}=0.027)$.
Twenty one days after the SCI, the BBB score in 5 rats from the $30 \mathrm{~g}$ compression (subgroup-1) was $8-9$ points (an average value of 8.4 points), in the $40 \mathrm{~g}$ subgroup- 2 , it was $3-7$ points (an average value of 5.8 points) and in $50 \mathrm{~g}$ subgroup-3, it was $2-4$ points (average 3.0 points). The statistical analyses of the results showed a highly significant difference in the improvement of the motor functions between rats belonging to the subgroup-1, versus rats belonging to the subgroup-3 $(\mathrm{P}=0.0001)$, a significant difference between rats belonging to the subgroup-2, versus rats belonging to the subgroup-3 $(\mathrm{P}=0.0062)$ and a significant difference between rats belonging to the subgroup-1 versus rats belonging to the subgroup-2 $(\mathrm{P}=0.01)$.

Twenty eight days after the SCI, the BBB score in 5 rats from the subgroup- 1 was $8-10$ points (an average value 9.0 points), in subgroup-2, it was $3-8$ points (an average value 6.2 points), and in subgroup-3 it was $3-5$ points (average 3.8 points). The statistical analyses showed a highly significant difference between rats belonging to the subgroup-1, versus rats belonging to the subgroup-3 $(\mathrm{P}=0.0002)$, a significant difference between rats belonging to the subgroup-2, versus rats belonging to the subgroup-3 $(\mathrm{P}=0.02)$, and a significant difference between rats belonging to the subgroup-1, versus rats belonging to the subgroup-2 $(\mathrm{P}=0.003)$.

The behavioural outcomes expressed as the BBB scores are presented in Graph 1. The significant intergroup differences were found at all survival times longer than 7 days post SCI.

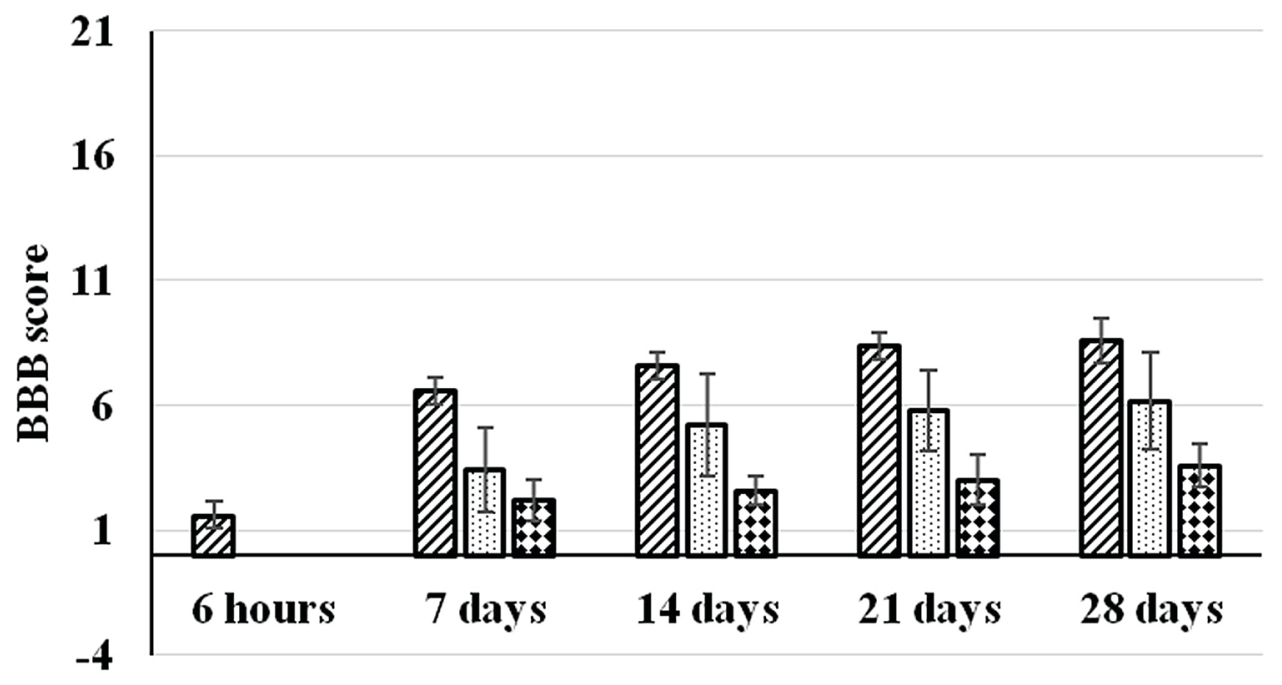

\section{$\nabla 1$ subgroup $\quad \mathbf{2}$ subgroup $\$ 3$ subgroup}

Graph 1. Comparison of the averaged data of locomotor rating scale for open field testing obtained in rats from three experimental subgroups 


\section{DISCUSSION}

More than a decade ago, spinal cord traumatic lesions meant for patients, the confinement to a wheelchair and different medical complications for the rest of their lives [3], [5], [18]. The treatment armamentarium was limited and the provision of care for individuals with SCI was, in a majority of cases, met with frustration [3], [5], [18]. Progress in neuroscience research has made the idea, that SCI will eventually be repairable, plausible [12], [13]. The use of animal models is critical for devising experimental therapies aimed to restore functions lost due to SCI [14]. Animals such as rabbits, cats, dogs, swine (especially minipigs), non-human primates, as well as rats and mice, are used in different experimental models [8], [9], [14-17], [19], [20]. Due to their cost, accessibility, ease of care and the existence of well-established functional analysis techniques, rat experimental models are the most commonly used at present [2], [6], [15], [19]. At the Institute of Neurobiology, Slovak Academy of Sciences, where we performed our study, they have been breeding Wistar rats for a long time. We decided to use adult female rats of this strain in our experiments.

Different types of SCI protocols address different purposes, therefore each experimental model has its own advantages and disadvantages.

The transection model requires durotomy and partial or complete cutting of the spinal medulla. A lateral hemisection is sometimes preferred because the integrity of one half of the spinal cord is sufficient to maintain urinary bladder and bowel function, and results in less intense postoperative care and an increased animal survival rate. The transection models provide valuable information when axon regeneration is studied or implantation of specific devices are tested. The disadvantage of this SCI model is that transection of the spinal medulla is rarely seen in human patients [3], [7], [18].

The SCI in the contusion model, is induced by hitting the spinal medulla through the exposed dura mater with a blunt contusion force, with a weight dropped from a defined height or with a spinal cord injury computer operated apparatus [20]. These devices allow the measurement of biomechanical parameters such as; impulse, velocity, power and energy. The contusion type of injury better mimics the lesions seen in humans than the transection models [11, 20]. However, it is more difficult to distinguish the spared and regenerated tissues in this model [6].
The SCI in the compression models are induced by compressing the spinal medulla with an aneurysm clip, forceps, balloon or an impactor acting through the intact dura mater. By the help of this model, it is possible to create different degrees of SCI by adjustment of the compression strength and time [19]. The compression model also mimics the neuropathology observed in humans [11]. The disadvantages of compression models are problems with securing reproducibility. The compression force of an aneurysm clip diminishes with each application and every clip closes with a different strength. The compression force of an intraspinally inserted balloon inflated by the exact volume of solution, changes slightly depending upon the diameter of the vertebral canal utilized. We have overcome these problems by utilisation of an impactor compressing the spinal medulla by a defined weight for a defined time interval.

The key element of any SCI study is the ability to acquire reliable, reproducible and worthwhile behavioural data [9], [15], [19]. The most commonly used test to assess motor functions after a SCI is the 21 point BBB locomotor scale [2]. Each point in the BBB score represents a specific set of characteristics of pelvic limb function after thoracic or thoracolumbar spinal cord trauma demonstrated by the rat during spontaneous open field locomotion [2]. We decided to use the assessment of the BBB score in our study.

The goal of all SCI models is to produce submaximal injury, where the outcome can be measurably improved or deteriorated by the therapeutic intervention of interest. The statistical analyses of the data gained in three experimental subgroups demonstrated that these requirements were achieved most reliably in the subgroup- 2 of rats following the $40 \mathrm{~g}$ compression spinal cord trauma.

\section{CONCLUSIONS}

This study confirmed the reproducibility and reliability of the rat spinal cord compression model. The behavioural characteristics expressed by the BBB scores were similar to the consequences of medullary trauma in different experimental animals as well as in humans. The model proved its suitability for preclinical testing of new therapeutic approaches aimed to modulate neuropathological processes participating in secondary injury mechanisms and to improve the outcome in para- or tetraplegic patients. 


\section{REFERENCES}

1. Animal Protection Act of the Slovak Republic No. 15/1995, part 39 (In Slovak), 1250-1255.

2. Basso, M.D., Beattie, M.S., Bresnahan, J.C., 1995: A sensitive and reliable locomotor rating scale for open field testing rats. J. Neurotrauma, 12, 1-21.

3. Beneš, V., 1987: Spinal Cord Injuries (In Czech), 3rd edn., Avicenum, Prague, 189 pp.

4. Dolan, I., Šrámková, T., 2013: Spinal cord injuries, incidence in Slovakia and prevention. Via Pract., 10, 203-205.

5. Dryden, D. M., Saunders, L. D., Jacobs, P., Schnopflocker, D. P., Rowe, B.H., May, L.A., et al., 2005: Direct health care costs after traumatic spinal cord injury. J. Trauma, 59, 443-449.

6. Lee, D. H., Lee, J. K., 2013: Animal models of axon regeneration after spinal cord injury. Neurosci. Bull., 29, 436-444.

7. Lee, B. B., Cripps, R. A., Fitzharris, M., Wing, P. C., 2014: The global map of traumatic spinal cord injury epidemiology: Update 2011, global incidence rate. Spinal Cord, 52, 110-116.

8. Maženský, D., Petrovová, E., Luptáková, L., 2011: Variations of the origin of arteries supplying the brain in the rabbit and rat ischemia experiments. Folia Veterinaria, 55, 133-136.

9. Navarro, R., Juhas, J., Keshavarzi, S., Juhasova, J., Motlik, J., Johe, K., et al., 2012: Chronic spinal compression model in minipigs: A systematic behavioural, qualitative, and quantitative neuropathological study. J. Neurotrauma, 29, 499-513.

10. Popesko, P., Rajtová, V., Horák, J., 1990: The Atlas of Anatomy of Small Laboratory Animals (In Slovak). 2nd edn., Príroda, Bratislava, 253 pp.

11. Oyinbo, C.A., 2011: Secondary injury mechanisms in traumatic spinal cord injury: A nugget of this multiply cascade. Acta Neurobiol. Exp., 71, 281-299.
12. Rossignol, S., Schwab, M., Schwartz, M., Fehlings, M. G., 2007: Spinal cord injury: Time to move? J. Neurosci., 27, 11782-11792.

13. Rowland, J. W., Hawryluk, G. W. J., Kwon, B., Fehlings, M. G., 2008: Current status of acute spinal cord injury pathophysiology and emerging therapies: Promise on the horizon. Neurosurg. Focus, 25, 1-17.

14. Silva, N.A., Susa, N., Reis, R.L., Salgado, A. J., 2014: From basics to clinical: A comprehensive review on spinal cord injury. Progr. Neurobiol., 114, 25-57.

15. Stokes, B. T., Jakeman, L. B., 2002: Experimental modelling of human spinal cord injury: A model that crosses the species barrier and mimics the spectrum of human cytopathology. Spinal Cord, 40, 101-109.

16. Šulla, I., jr., Maršala, J., Šulla, I., 2003: Study of schemic and compressive paraplegia in dogs. In 12th European Congress of Neurosurgery, Lisbon, Portugal, September 7-12, Monduzzi Editore, International Proceedings Division, Bologna, 891-894.

17. Šulla, I., Bačiak, L., Juránek, I., Cicholesová, T., Boldižár, M., Balik, V., Lukáčová, N., 2014: Assessment of motor recovery and MRI correlates in a porcine spinal cord injury model. Acta Vet. Brno, 83, 393-397.

18. Tchvaloon, E., Front, L., Gelernter, I., Ronen, J., Bluvshtein, V., Caty, A., 2008: Survival, neurological recovery and morbidity after spinal cord injuries following road accidents in Israel. Spinal Cord, 46, 145-149.

19. Vanický, I., Urdzíková, L., Saganová, K., Čížková, D., Gálik, J., 2001: A simple and reproducible model of spinal cord injury induced by epidural balloon inflation in the rat. J. Neurotrauma, 18, 1399-1407.

20. Vijayaprakash, K.M., Srindharan, N., 2013: An experimental spinal cord injury rat model using customized impact device: A cost-effective approach. J. Pharmacol. Pharmacother., 4, $211-213$

Received April 25, 2016 